\title{
Changing Trends in Computer Architecture : A Comprehensive Analysis of ARM and x86 Processors
}

\author{
Khushi Gupta;, Tushar Sharma \\ UG Student, School of Computer Science and Engineering, Poornima University, Jaipur, Rajasthan, India
}

Article Info

Volume 7, Issue 3

Page Number: 619-631

Publication Issue :

May-June-2021

\section{Article History}

Accepted : 20 June 2021

Published : 30 June 2021

\section{ABSTRACT}

In the modern world, we use microprocessors which are either based on ARM or x86 architecture which are the most common processor architectures. ARM originally stood for 'Acorn RISC Machines' but over the years changed to 'Advanced RISC Machines'. It was started as just an experiment but showed promising results and now it is omnipresent in our modern devices. Unlike x86 which is designed for high performance, ARM focuses on low power consumption with considerable performance. Because of the advancements in the ARM technology, they are becoming more powerful than their x86 counterparts. In this analysis we will collate the two architectures briefly and conclude which microprocessor will dominate the microprocessor industry. The processor which will perform better in different tests will be more suitable for the reader to use in their application. The shift in the industry towards ARM processors can change how we write softwares which in turn will affect the whole software development environment.

Keywords: ARM (Advanced RISC Machine), ISAs (Instruction Set Architectures), RISC (Reduced Instruction Set Computing), CISC (Complex Instruction Set Computing), Instruction Set, Data visualization

\section{INTRODUCTION}

Microprocessors are present everywhere in the modern world from smartphones, to smart cars and without them the world would be a totally different place. Modern technology allows us to achieve the quality of life we have today and the technological marvel. Over the years the processing power of processors have increased dramatically with less power consumption. But as we take a deeper look into microprocessors, we will notice that there are mainly two architectures which are dominant in the industry. In the research paper we will take a deeper look into the working of both the types. But the basic difference between ARM and x86 is the ideology on how to tackle a mathematical problem. $x 86$ chose the more complex approach to appeal to the business for their heavy use. But ARM chose to go with a much more simple approach by involving the necessary operations. Apple recently launched their M1 processor series which shocked the whole industry due to its performance. This difference led to the two totally different approaches for microprocessor 
architectures. We will discuss the difference further in the research paper.

In this research paper we will take a deeper look into both the processors by comparing the different parameters. This comparative analysis involves different parameters on which both the processors are compared and a conclusion is drawn. The comparison includes power consumption, total throughput, softwares supported, processing power, applications, and supported technologies. The comparisons are made on the basis of real data compiled and formed in the tables and graphs. Data visualization is used to better make our point understood to the reader and show how both the processors differ. ARM and x86 have similar performance in their latest processors with some minor changes such as power consumption, heat generation, and more. These differences led to a massive advantage on ARM's side as showcased by the latest Apple's M1 series. The new M1 not only performed better but also did it while having half the power consumption. When such results are given by an ARM chip which was considered only for mobile use, we need to see what led to these differences.

There are perpetual improvements to both processors which implies that what's great now may not be so great in a year. With the M1 chip from Apple thumping the market in 2021, the company vindicates that this ARM chip will produce twice the power for one-third of the battery consumption (Graham-Smith, 2021).

\section{A. Challenges to Intel Processors}

The fazing challenges Intel needs to address are:

- Manufacturing dilemma

- Lost opportunity in IoT and mobile

- $\quad$ Risky opportunities in edge computing

- Declining data center volumes and margins

- Voracious appetite for AI compute
- Opportunities abound in programmable processors

- Geopolitical tensions

The remaining paper is organized as follows. Section 2 provided connected run through literature survey. Section 3 demonstrates material and ways of this analysis. Section 4 describes our experimental setup and methodology, and Section 5 presents the analysis of our findings and data. Finally, the conclusion of the research is stated in section 6 .

\section{LITERATURE REVIEW}

In order to justify the research various already defined papers, articles were studied. Blem and Menon [1] in their research paper have analysed both the x86 and ARM architecture in depth. The aim behind the research paper is to revisit the debate of which of the following architectures is best in the modern-day scenario with people using more and more complex softwares. With the growth in workload of modern systems the ISA plays a huge role in power consumption, throughput of the system, heat production, and efficiency. The argument is supported by total bit tables, performance graphs, execution tables, similarity, and comparative tables. This data gives the reader a clearer idea on the difference between both the architectures and lets them support their argument with real data. This paper also includes the working of both the architectures which gives ideas about system challenges and encountered tables.

Rafael et al. [2] in his research paper moves towards the practical application and feasibility of servers which utilizes the modern ARM computer architecture and then compares the experimental results with those of the current servers with x86 computer architecture. The research paper focuses on the question of which is the following computer architecture is more suited for serves and in which 
circumstances the ARM processor is preferred over the traditional x86 servers. The difference and similarities are argued based on the comparison execution on web and server applications. Variables such as number of requests, temperature, power consumption, head generation, request latencies, and stability are considered and included in the final data. The research paper provides a more in-depth capabilities of the ARM computer architecture on the server side and how it can affect the working of the current servers by providing more performance with low operation costs.

H. El-Aawar [3] in his research he compared the processing capabilities of ARM and x86 processors and a conclusion is made on the basis of total energy consumed e, throughput, average cycles per instruction (CPI), and L2 cache miss rate. Both the processors have been competitors for a long time but they are mainly in different segments. But with the advancement in the ARM technology, the performance is close to the mainstream $\mathrm{x} 86$ processors. To compare both the processors, the Gem5 computer simulator is used which again and again shows that ARM outperforms the x86.

M. Rebaudengo et al. [4], their paper deals with a method to provide a microprocessor-based system with safety capabilities. The method exploits a set of transformations which can automatically be applied, thus greatly reducing the cost of designing a safe system, and increasing the confidence in its correctness. Fault Injection experiments have been performed on a sample application using two different systems based on CISC and RISC processors.

Mohak Chadha et al. [5], their research paper uses PMC (Performance Monitoring Counters) to build accurate power models which is a more statistical approach for $\mathrm{x} 86$ processor analysis. The results from the tests are supported by sophisticated reference measurements along with $\mathrm{k}$-fold cross validation technique. To put processors under heavy loads, synthetic workload is generated and then the results are monitored. With the help of this synthetic load the measurements are taken and results are concluded.

Sirin et al. [6], takes a more practical approach by comparing ARM Cortex-A57 with a high performing Intel $\mathrm{x} 86$ processor with parameters such as latency quantified, power consumption, and throughput delivered. After running several tests the conclusion is made that ARM processors consume 3-15 times less power all the while being upto 9 times energy efficient. However, the throughput when compared to $\mathrm{x} 86$ processors is $1.7-3$ times lower and the quantified latency can be upto 11 times higher. This suggests that all thought ARM processors use less power but when used for servers, the performance is reduced significantly with high latency.

Otto et al. [7], use cross-layer optimization on x86 and ARM in their research paper to see any chance in energy efficiency, power usage, performance with different frequency settings, compiler, voltage, multithreading strategies, and more. The measurements are taken and conclusion is drawn with the help of single core vs multi core, corner detections, compiler level, real-time performance constraints, and crosslevel optimization.

N. Stephens et al. [8], in their research paper takes a dive in the promising ARM processors and their $5 \mathrm{G}$ networking capabilities along with other advantages. The research paper is focused on the latest Neoverse N1 platform which offers a scalable portfolio for high efficiency machines.

Davi et al. [9], dicusses randomization solution, called Xifer, that disperses all code across the whole address space, re-randomizes the address space for each run, is compatible to code signing, and does neither require offline static analysis nor source-code in their research paper.

Kristopher Keipert $\dagger$ et al. [10], their research paper compares the computational order with the GAMESS quantum chemistry suite; the $\mathrm{x} 86$ system is compared with the 32-bit and 64-bit ARM system. When 
benchmark scores are compared it is noticed that $\mathrm{x} 86$ performs every computation much faster compared to 32 and 64-bit ARM processors. The results are similar to Hartree-Fock and density functional theory energy calculations which was the expected result. However, ARM performed much better for memory-intensive second-order perturbation theory energy and gradient computations, where x86 took $86 \%$ longer than the 64-bit ARM processor. When it comes to power consumption, the 32-bit ARM processor is more efficient than the $\mathrm{x} 86$ processor for the same number of core count and molecular count.

D. Patterson (Patterson, 2017), in his article described the reduced instruction set computer (RISC) as an alternative to the general trend at the time toward growth of complex instruction sets. It concludes that RISC executes most instructions in a single short cycle.

Nicholas et al., in their paper comes up with a brief survey of different hardware/software security attacks and summarizes a differences of security features in RISC-V and other traditional architectures along with security extensions that can be achieved by RISC-V.

Other research papers focus on the theoretical approach and tests which may or may not indicate the real world differences in both the processors which is important in the end. Also the Apple's M1 chip is not taken into consideration which is the first user grade microprocessor in a laptop. The M1 was under development for a long time and it changed the industry standards and what users expect their laptops or desktops to perform. There is not a lot of research and comparison done in the M1 chips which showcase the true potential of the ARM based processors on a laptop with much more work loads.There have already been many research papers with comparisons of both the processors but the softwares used to show the performance difference did not reflect the real world scores or difference.
The research paper picks up where the other papers lag and where new processor technology came into play for the general public. It shows how the M1 outperforms x86 processors in almost all the areas such as power consumption, performance, heat reduction, and more.

Comparing only CISC and RISC is not enough to make a strong argument that whether ARM or x86 is better for any given task. Other research papers deal with the ARM implementation in other areas such as servers and backend devices which does not give ARM the advantage of low power consumption since servers are expected to use more power and does not benefit from less power consumption since the main focus is on performance only. Laptops equipped with the ARM based processors show how the consumer grade devices can benefit by shifting from the traditional $\mathrm{x} 86$ to the new ARM based processors.

Based on the literature survey and identified research gaps, the following research objectives are identified. The main idea behind the research is "What are ARM and x86 processors, and Are they going to replace x86 (Intel)?". Secondly "Which processor to use when and in which type of operating systems?".

There have already been many researches comparing two Processors. But it is also important to compute the comparative analysis of the variants of ARM and x86 processors. The research that has already taken place was all related specifically based on comparison of ISA. That is, ISA plays an important role in performance, power and energy efficiency or only comparison of RISC and CISC. This paper would compare the two of them as well along with the performance of the different processors. The research papers on comparative analysis are more oriented towards older implementations of the ARM processors but do not cover the latest M1 processors which are specifically made by Apple to outperform x86. The M1 processor was under development for a 
long time and takes inspiration from Apple's A series of mobile processors. Both the A series and M1 series of processors are made in house by Apple which puts them ahead of the competition and leaves other microprocessor makers to play catch up. The introduction of laptop/desktop grade ARM processors is a very significant event to not be taken into account when comparing with the traditional x86 which are made specifically for laptop/desktop. The results show a trend of ARM processors outperforming every x86 processor, however processors made for servers or desktops are not taken into consideration since they are not covered under laptop processors.

\section{RESEARCH METHODOLOGY}

The purpose of this study is to show the difference in the two most popular processor technologies and which of the two can overtake the industry in the coming years. To make the comparison we will run benchmarks on different systems with both the processors and then compile the data into graphs and tables. We will also look into the working of both the processors and why they are used for different applications. The workings of both the processors are kept in mind and multiple benchmarks are taken to get the fair comparison. This study compares two of the most popular processors and concludes which of them will be widely adopted in the coming years. The processor will determine the future of softwares used and how it will affect the whole industry. We will be looking at real data from different benchmarks and comparing them with different parameters. But before we can get started with the comparisons, we need to know about both the processors.

\section{A. RISC vs CISC}

Now the question arises as to why there is such a huge difference in the power consumptions of both the processors. The difference arises due to different
ISAs(Instruction Set Architectures), you can understand it as different methods of handling the same problem. The output will be the same but the way in which we handle a mathematical problem will be different. This difference in the ISA affects how many instruction cycles will be performed for any given task affecting the power usage, performance, memory usage, heat generation, and more. Now we should see which are the main two ISAs that we use in our modern microprocessors.

In the ARM processor we use the RISC (Reduced Instruction Set Computing) which as the name suggests will use more compartmentalization(categorized) in simpler instruction sets making the overall work much more simpler, meaning less clock cycles. Simpler instructions for the processor to perform means that we need fewer transistors on our physical circuit board, making it consume less power. This is the reason why ARM chips are much more efficient and smaller in size making them ideal for smaller portable devices. However, more memory is needed to perform the same task due to rise in the number of operations which increase the execution time. This is the reason why multiprocessors programming is important in the software part to get the most out of the processor and avoid putting all the load on our main thread(one processor).

On the other hand, x86 uses the CISC (Complex Instruction Set Computing) which as the name suggests is more oriented using more clock cycles for performing the same task. Due to the increased number of clock cycles, the processor will have more transistors producing more heat and consuming more power. The idea behind CISC is to have the maximum output from the processor and it is not concerned about the power usage. When compared to RISC, CISC is more capable when it comes to heavy tasks (complex mathematical instructions) which makes the work of the compiler much more 
simpler. We do not need to divide the work between different processors unless the task is very big. On the software side, the logic of the programs will be much more simpler. This is the reason why we do not use the x86 processor for hand held devices, this is why the MacBooks with the M1 processor (ARM) have double the battery life when compared to x86 based laptops. Now we should look at both the processors in depth to understand them better before we can start with the comparison.

\section{B. $x 86$ - The Powerhouse}

Intel uses the $\mathrm{x} 86$ architecture due to the high raw processing power capabilities that is very appealing for desktop users and to be used in servers. The x86 processor uses 64-bit computing which supports a variety of different operating systems like Windows, Linux, MacOS. The $\mathrm{x} 86$ is more oriented towards handling the complex mathematical operations and tasks. But due to the increased number of operations (mathematical operations) the power usage is much higher than its counterpart. The x86 processor consumes anywhere from $5-30$ Watts however, ARM processors take less than 5 Watts. This hugely benefits the hand-held devices such as smartphones, tablets, laptops, etc.

\section{ARM - The Efficiency Expert}

There was no real competition in the market after Intel mastered their design, and the ARM was started as a possible alternative. ARM (Advanced RISC Machines) is the most used microprocessor after the invention of handheld personal phones since it uses a lot less power. Modern companies like Samsung, Qualcomm, MediaTek, Apple use this architecture to design their own microprocessor. The focus of the ARM processor is low power consumption which enables most of the modern IOT devices. Unlike x86, ARM uses both the 32-bit and the 64-bit architectures depending on the requirement of the device maker. Over time the performance of the
ARM processors increased drastically as the processor is in its initial stage unlike the x86. The latest M1 processor based on ARM is capable of outperforming the $\mathrm{x} 86$ which uses a fraction of the power. Aside from portable devices, the ARM processor is also in the Mac 2021 (desktop). Apple is the industry leader and other companies adapt their ways and changes they make, this is why it is possible that $\mathrm{x} 86$ soon can become obsolete. However, each microprocessor is designed specifically for different Operating Systems.

\section{EXPERIMENTAL SETUP}

For our measurements we are using different systems(laptop) with similar performance and are equipped with the best $\mathrm{ARM}$ and $\mathrm{x} 86$ processors.

\section{A. Configurations of samples taken}

1) M1 MacBook Pro 13"

$>\mathrm{CPU}$ cores -8

$>$ Performance cores -4

$>$ Efficiency cores -4

$>$ GPU cores -8

$>$ Neural Engine cores -16

2) Intel MackBook Pro 13"

$>1.4 \mathrm{GHz}$ quad-core 8 th-generation Intel Core i5 with Turbo accelerate to $3.9 \mathrm{GHz}$ or $2.0 \mathrm{GHz}$ quad-core

$>$ 10th-generation Intel Core i5 with Turbo accelerate to $3.8 \mathrm{GHz}$

3) M1 MacBook Air

$>\mathrm{CPU}$ cores -8

$>$ Performance cores -4

$>$ Efficiency cores -4

> GPU cores -7

$>$ Neural Engine cores - 16

4) Intel M1 MackBook Air 
1.1GHz quad-core Intel Core i5, Turbo accelerate to $3.5 \mathrm{GHz}$, with $6 \mathrm{MB} \mathrm{L} 3$ cache

\section{B. Softwares used}

Various different softwares are used for benchmarking which are commonly used to test capabilities of each processor. We conclude the section with discussing the results obtained from the test and what it tells about the processor capabilities. The measurements are taken multiple times to average out a consistent score and are compared with other systems. All the systems are given ideal conditions to provide the highest score possible without overclocking the processors for higher unstable scores.

Let's have the glance at the Benchmark scores of Apple M1 vs Intel -

\section{1) GeekBench 5}

In the GeekBench 5, the M1 is the speedy processor and has a 1,730 single core score. Intel has a 1,349 score. And in the multi-core test, Apple M1 glints with a score of 7,578 and is even superior to Intel.

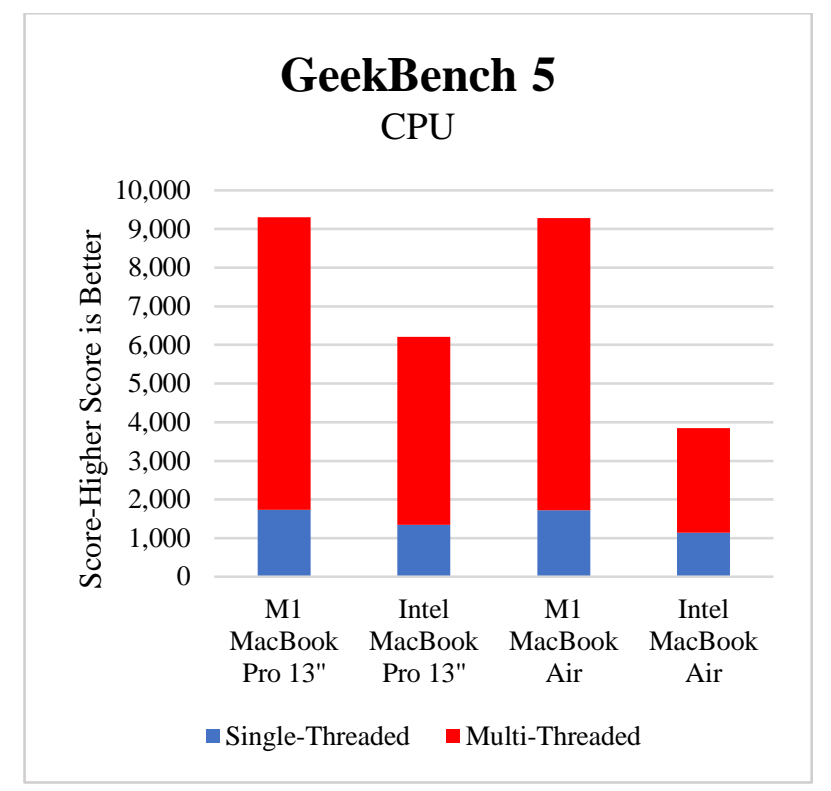

Figure 1. GeekBench 5 Single and multi-core score analysis
TABLE I

GEEKBENCH 5 SCORES

\begin{tabular}{|l|l|l|l|l|}
\cline { 2 - 5 } \multicolumn{1}{c|}{} & $\begin{array}{l}\text { M1 } \\
\text { MacBook } \\
\text { Pro 13, }\end{array}$ & $\begin{array}{l}\text { Intel } \\
\text { MacBook } \\
\text { Pro 13, }\end{array}$ & $\begin{array}{l}\text { M1 } \\
\text { MacBook } \\
\text { Air }\end{array}$ & $\begin{array}{l}\text { Intel } \\
\text { MacBook } \\
\text { Air }\end{array}$ \\
\hline $\begin{array}{c}\text { Multi- } \\
\text { Threaded }\end{array}$ & 7,578 & 4,864 & 7,565 & 2,716 \\
\hline $\begin{array}{c}\text { Single- } \\
\text { Threaded }\end{array}$ & 1,730 & 1,349 & 1,720 & 1,136 \\
\hline
\end{tabular}

2) Cinebench R23

In the below graph intel sample offers a maximum score of 1,149 on the single core. On the other hand, the M1 score is 1,494 . For the multicore score, M1 scores 7,791 , that is actually faster than intel which scores 5,208 .

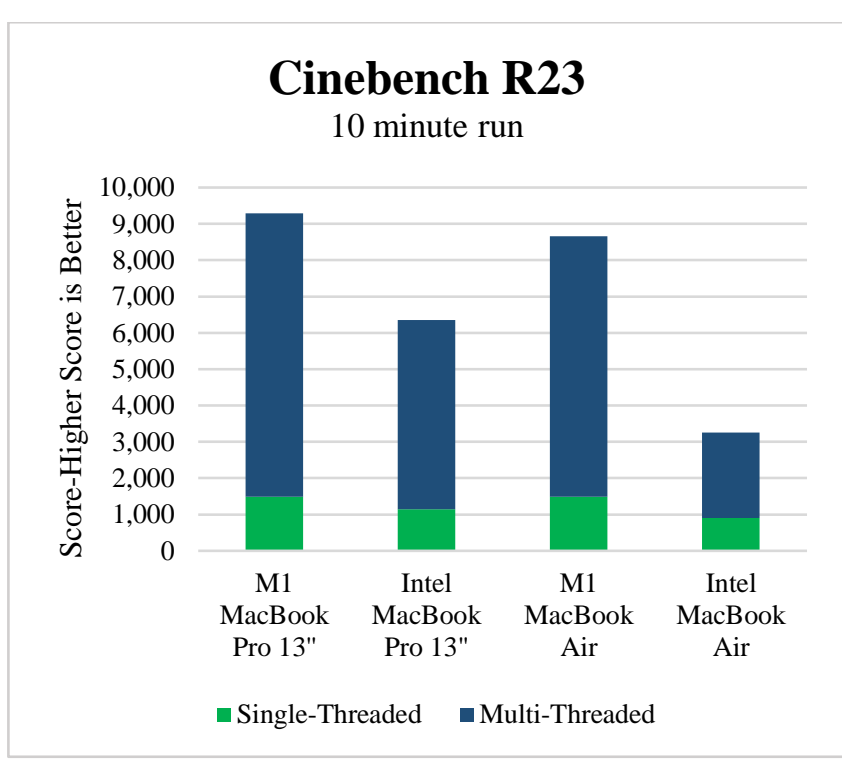

Figure 2. Cinebench R23 Single and multi-core score analysis

TABLE I

CINEBENCH R23 SCORES 


\begin{tabular}{|l|l|l|l|l|}
\cline { 2 - 5 } \multicolumn{1}{c|}{} & $\begin{array}{l}\text { M1 } \\
\text { MacBook } \\
\text { Pro 13', }\end{array}$ & $\begin{array}{l}\text { Intel } \\
\text { MacBook } \\
\text { Pro 13', }\end{array}$ & $\begin{array}{l}\text { M1 } \\
\text { MacBook } \\
\text { Air }\end{array}$ & $\begin{array}{l}\text { Intel } \\
\text { MacBook } \\
\text { Air }\end{array}$ \\
\hline $\begin{array}{c}\text { Multi- } \\
\text { Threaded }\end{array}$ & 7,791 & 5,208 & 7,168 & 2,362 \\
\hline $\begin{array}{c}\text { Single- } \\
\text { Threaded }\end{array}$ & 1,494 & 1,149 & 1,490 & 899 \\
\hline
\end{tabular}

3) Adobe Creative Cloud

Adobe creative cloud showcases the practical use case which is much more helpful when compared to benchmark softwares, unfortunately the data for Intel counterpart was not available.

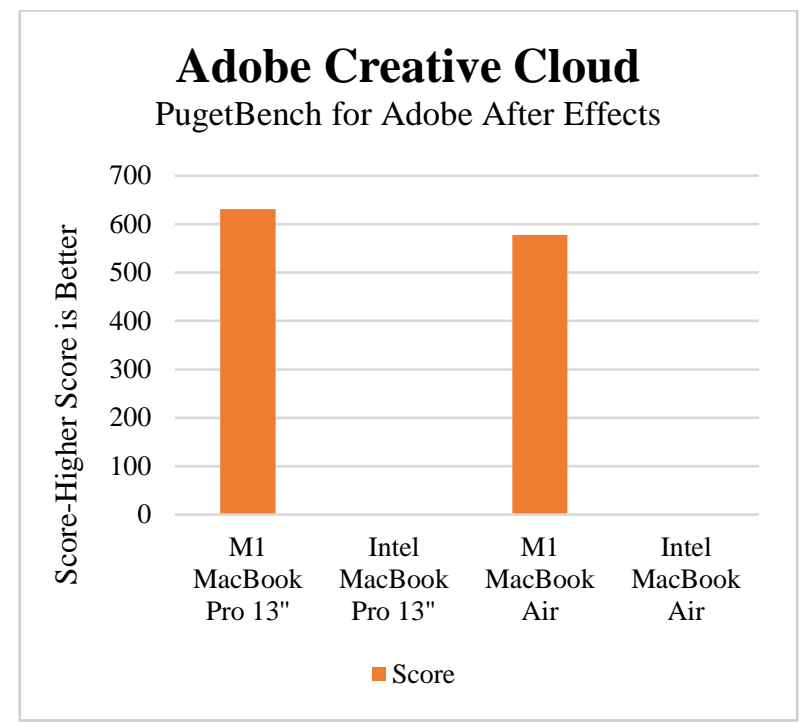

Figure 3. Adobe Creative Cloud score analysis

TABLE IIII

ADOBE CREATIVE CLOUD SCORES

\begin{tabular}{|c|l|l|l|l|}
\cline { 2 - 5 } \multicolumn{1}{c|}{} & $\begin{array}{l}\text { M1 } \\
\text { MacBook } \\
\text { Pro 13, }\end{array}$ & $\begin{array}{l}\text { Intel } \\
\text { MacBook } \\
\text { Pro 13, }\end{array}$ & $\begin{array}{l}\text { M1 } \\
\text { MacBook } \\
\text { Air }\end{array}$ & $\begin{array}{l}\text { Intel } \\
\text { MacBook } \\
\text { Air }\end{array}$ \\
\hline $\begin{array}{c}\text { Single- } \\
\text { Threaded }\end{array}$ & 631 & 0 & 578 & 0 \\
\hline
\end{tabular}

\section{4) Handbrake 1.4.0}

Handbrake is an open source video transcoder which gives us actual performance of each processor and how they would perform in the real world. We have to note that native support for the M1 processors is not available due to which the performance was affected. The M1 processors finish the encoding in under 4 minutes and 30 seconds whereas Intel takes over 6 minutes. This gives us an idea about the processing capabilities of the ARM architecture.

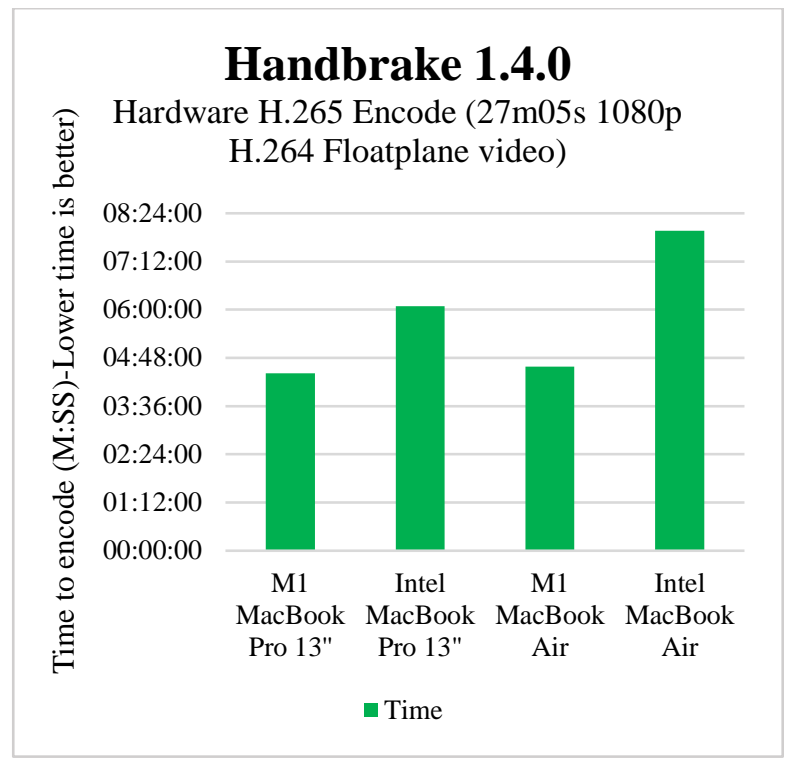

Figure 4. Handbrake 1.4.0 time to encode analysis

TABLE III

HANDBRAKE 1.4.0 TIME TO ENCODE

\begin{tabular}{|l|l|l|l|l|}
\cline { 2 - 5 } \multicolumn{1}{c|}{} & $\begin{array}{l}\text { M1 } \\
\text { MacBook } \\
\text { Pro 13, }\end{array}$ & $\begin{array}{l}\text { Intel } \\
\text { MacBook } \\
\text { Pro 13, }\end{array}$ & $\begin{array}{l}\text { M1 } \\
\text { MacBook } \\
\text { Air }\end{array}$ & $\begin{array}{l}\text { Intel } \\
\text { MacBook } \\
\text { Air }\end{array}$ \\
\hline $\begin{array}{c}\text { Cingle- } \\
\text { Threaded }\end{array}$ & $04: 25: 00$ & $06: 05: 00$ & $04: 35: 00$ & $07: 58: 00$ \\
\hline
\end{tabular}

When compared to the $\mathrm{x} 86$ Intel processors, the M1 processors performed better when processors were involved. Outperforming the Intel which uses significantly more power and produces more heat which depletes the battery much faster and reduces the efficiency (drop in clock speed).

\section{ARM vs Intel x86 Processors on Cloud Platform}

The growth of cloud computing and infrastructureas-a-service (IaaS) resist hope for energy-efficient architectures such as ARM. Since energy 
consumption by servers forms the major fraction of the operational cost for cloud data centers, ARM with its lower energy spoor and server-grade memory addressing has started to become a viable platform for servers hosted by Cloud providers. This is particularly captivating given that scale-out workloads are common to Cloud applications, and the growing trend of containerization as opposed to virtualization. For analysis we have taken CPUs of 2, 4 and 8 cores with ARM and x86 processors and then analyse the cost of the respective processors for different cloud services i.e. Microsoft azure and Amazon Web Services (AWS).

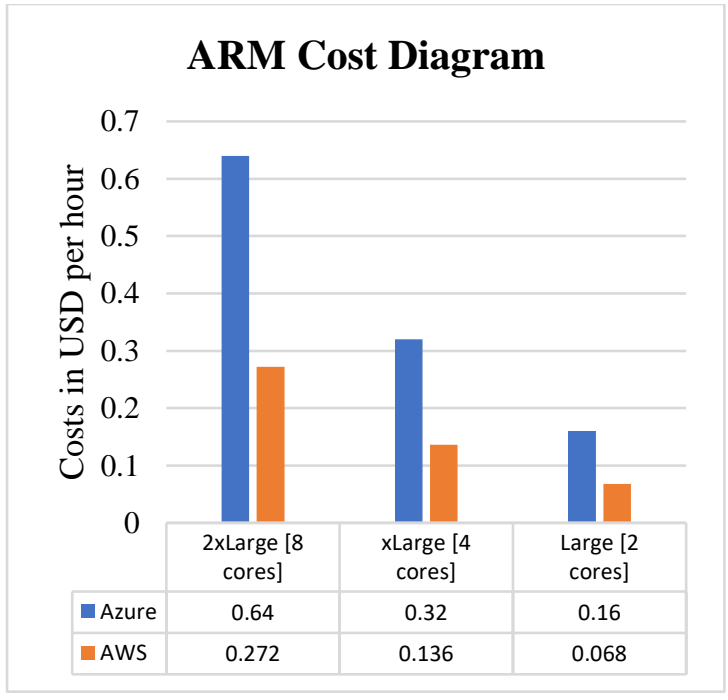

Figure 5. Cost analysis for ARM instances

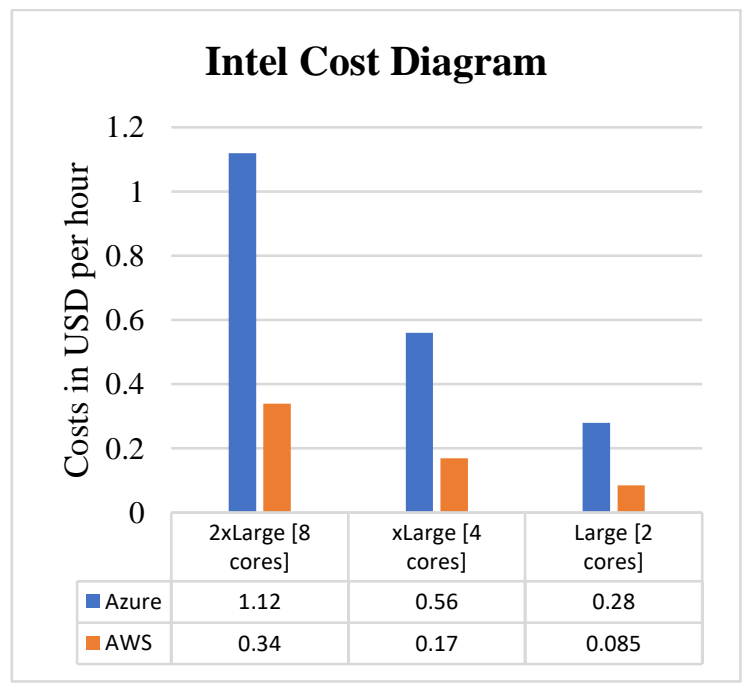

Figure 6. Cost analysis for Intel instances
TABLE V

COMPARISON OF COSTS OF PROCESSORS ON CLOUD PLATFORM

\begin{tabular}{|l|l|l|l|l|}
\hline \multirow{2}{*}{$\begin{array}{l}\text { Cloud } \\
\text { Services }\end{array}$} & \multicolumn{2}{|l|}{ ARM Cost } & \multicolumn{2}{l|}{ Intel Cost } \\
\cline { 2 - 5 } & Min & Max & Min & Max \\
\hline Azure & 0.16 & 0.64 & 0.28 & 1.12 \\
\hline AWS & 0.068 & 0.272 & 0.085 & 0.17 \\
\hline
\end{tabular}

For each instance form variation, we discovered that Intel processors were the most expensive, followed by ARM. As opposed to Intel, the results show that it can be $10 \%$ less expensive.

\section{RESULTS AND DISCUSSIONS}

We cannot keep using the widely adopted computer architecture and expect it to handle the increased workloads in the modern world, as the softwares are getting more demanding and complex. So, we need to select the best or the most preferable processor. We have analyzed the performance of different processors using different softwares and encoding time or the time for execution is measured. In order to choose the best processor, the performance and power are the major factors so here is a comparison of the ARM and $\mathrm{x} 86$ processors on specific parameters.

TABLE VI

COMPARISON OF PROCESSORS ON SPECIFIC PARAMETERS

\begin{tabular}{|c|c|c|}
\hline Parameters & $\mathrm{X} 86$ & ARM \\
\hline ISA & Uses CISC & Uses RISC \\
\hline RAM & $\begin{array}{lll}\text { Less } & \text { RAM is } \\
\text { used } & & \end{array}$ & $\begin{array}{l}\text { More RAM is } \\
\text { used }\end{array}$ \\
\hline Compiler & $\begin{array}{l}\text { Work done to } \\
\text { translate high- } \\
\text { level code is less }\end{array}$ & $\begin{array}{l}\text { Work done to } \\
\text { translate high- } \\
\text { level code is } \\
\text { more }\end{array}$ \\
\hline Power & $\begin{array}{l}\text { More power is } \\
\text { required }\end{array}$ & $\begin{array}{l}\text { Less power is } \\
\text { required }\end{array}$ \\
\hline
\end{tabular}




\begin{tabular}{|c|c|c|}
\hline $\begin{array}{l}\mathrm{H} / \mathrm{w} \text { and } \mathrm{S} / \mathrm{w} \\
\text { Requirements }\end{array}$ & $\begin{array}{l}\text { Hardware } \\
\text { requirement is } \\
\text { more but } \\
\text { software } \\
\text { requirement is } \\
\text { less }\end{array}$ & $\begin{array}{l}\text { Hardware } \\
\text { requirement is } \\
\text { less but } \\
\text { software } \\
\text { requirement is } \\
\text { more }\end{array}$ \\
\hline $\begin{array}{l}\text { Optimization } \\
\text { of } \\
\text { performance }\end{array}$ & $\begin{array}{l}\text { Software } \\
\text { approach }\end{array}$ & $\begin{array}{l}\text { Hardware } \\
\text { approach }\end{array}$ \\
\hline $\begin{array}{l}\text { Code } \\
\text { Expansion }\end{array}$ & $\begin{array}{l}\text { Code } \\
\text { is }\end{array}$ & $\begin{array}{l}\text { Managing code } \\
\text { expansion is } \\
\text { difficult }\end{array}$ \\
\hline Execution & $\begin{array}{l}\text { Time to execute } \\
\text { is more. }\end{array}$ & $\begin{array}{l}\text { Faster } \\
\text { Execution of } \\
\text { Instructions } \\
\text { reduces time. }\end{array}$ \\
\hline $\begin{array}{l}\text { Instruction } \\
\text { sets }\end{array}$ & $\begin{array}{l}\text { Requires more } \\
\text { transistors } \\
\text { (more hardware } \\
\text { space) }\end{array}$ & $\begin{array}{l}\text { Simple } \\
\text { instruction sets } \\
\text { require fewer } \\
\text { transistors (less } \\
\text { hardware } \\
\text { space) }\end{array}$ \\
\hline $\begin{array}{l}\text { Decoding of } \\
\text { Instructions }\end{array}$ & Complex & Easy \\
\hline Application & $\begin{array}{l}\text { Emplaced in } \\
\text { Laptops, Servers, } \\
\text { and Desktops } \\
\text { where high } \\
\text { performance } \\
\text { and stability } \\
\text { matters more }\end{array}$ & $\begin{array}{l}\text { Emplaced in } \\
\text { mobile devices } \\
\text { where size, } \\
\text { speed and } \\
\text { power } \\
\text { consumption } \\
\text { matters more }\end{array}$ \\
\hline
\end{tabular}

TABLE VII

OVERVIEW OF THE SUPPORT FOR VARIED OPERATING SYSTEMS FOR THESE 2 COMPETITORY ARCHITECTURES

\begin{tabular}{|l|l|}
\hline ARM & X86 \\
\hline Linux & Linux \\
Android & Android \\
Windows & Windows \\
Compact & Compact/Mobile \\
& Windows \\
\hline
\end{tabular}

By the below graph we can conclude that ARM (M1) processor is gives upto $2 \mathrm{x}$ faster CPU performance and matches peak PC performance using $25 \%$ of the power.

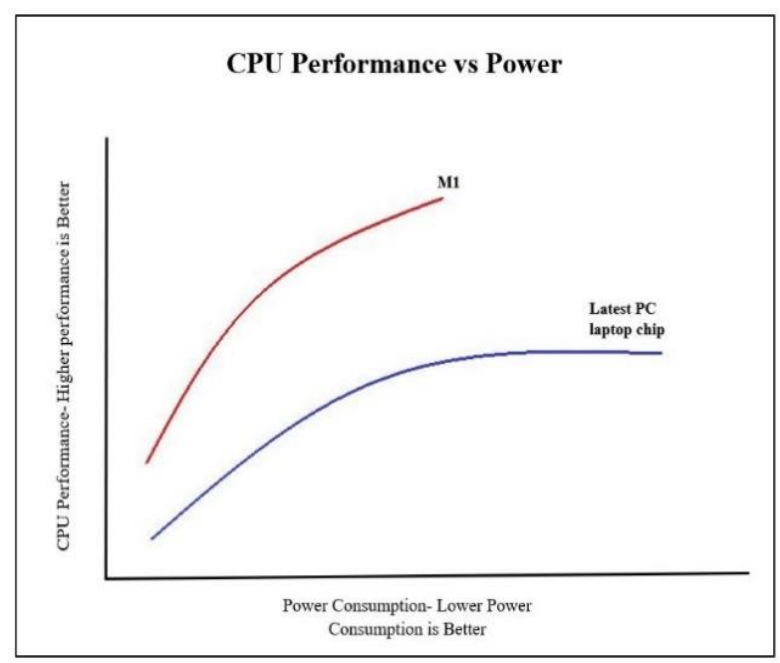

Figure 7. Graph of CPU Performance vs Power

\section{A. Key Findings 1}

The major findings from our benchmark analysis are:

- In some of the case there was no support for native softwares, despite it the M1 processors gave good performance.

- The benchmarks are comparable for both the x86 and ARM processors with the difference being in the power usage, RAM usage, and heat production.

\section{B. Key Findings 2}

The main findings from our analysis on cloud platform are:

- In public clouds like AWS, Arm-based frameworks and instances are readily accessible.

- AWS' Arm instances perform similarly to x86 instances in terms of computational performance.

- Arm instances are more cost-effective than x86 instances because they are considerably less expensive.

- Arm instances carry out better with "close to metal" applications. 


\section{Key Findings 3}

The M1 (ARM) processors outperformed the $\mathrm{x} 86$ processors in term of CPU power which is large performance gap considering the power usage was less than half and produced very low heat levels and the energy consumption is much less when simpler instruction cycles are used (RISC).

\section{Implications}

In our findings we find the trend of M1(ARM) processors being capable of outperforming every x86 processor available in the market by ADM and Intel. And due to simpler ISA the heat production is minimal which allows the less powerful M1 MacBook Air to work without a fan(heat displacement system) which is something not done in any x86 processor system. With the latest announcement from Apple about their Mac 2021 desktop series with ARM processors which are much smaller in size then their Intel counterpart and that much more powerful. Over time we expect the ARM processor to replace the $\mathrm{x} 86$ processor in virtually every IOT device due to its processing capabilities with lower power consumption. This shift in the processor technology can finally enable smaller IOT devices to become more capable and support a wide variety of features which are currently not possible with $\mathrm{x} 86$ processors.

\section{CONCLUSION}

In this research paper we have compared both the processors and their architectures which affect the working, performance, efficiency, and more. These results show which way the industry is heading and how we can expect the ARM processor to take over the $\mathrm{x} 86$. This will affect the technical capabilities of the IOT devices which will drastically change our lives. On the software side the industry will be more oriented towards App development occurring towards Android and IOS since there is a high possibility of Android taking over Microsoft. Suffice to say the gap between $\mathrm{x} 86$ and ARM is closing fast and has the capability to change the whole industry. Apple and other OEMs are competing to provide the best hardware which can handle more complex workload, thinner form factor, and more efficiency. With the introduction of the new M1 ARM based processor which gives Apple an edge over the competition. The M1 is very promising as it is more capable, all the while already widely used in handheld devices. With the integration of server technology with the ARM computer architecture can reduce the server costs all the while providing more output. This will directly affect our lives in one way or another with the introduction of more services and capabilities.

\section{REFERENCES}

[1]. E. Blem, M. Jaikrishnan and K. Sankaralingam, "Power struggles: Revisiting the RISC vs. CISC debate on contemporary ARM and x86 architectures," in 2013 IEEE 19th International Symposium on High Performance Computer Architecture (HPCA), 2013.

[2]. R. V. Aroca and L. M. Garcia Gonclaves, "Towards green data centers: A comparison of x86 and ARM architectures power efficiency," Journal of Parallel and Distributed Computing, vol. 72, no. 12, pp. 1770-1780, 2012.

[3]. H. El-Aawar, "CISC vs. RISC Hardware and Programming Complexity Measures of Addressing Modes," in Proceedings of the 2nd International Conference on Perspective Technologies and Methods in MEMS Design, 2006.

[4]. M. Rebaudengo, M. Reorda, M. Violante, P. Cheynet, B. Nicolescu and R. Velazco, "Evaluating the effectiveness of a software fault-tolerance technique on RISC- and CISCbased architectures," in Proceedings 6th IEEE 
International On-Line Testing Workshop (Cat. No.PR00646), 2000.

[5]. T. I. M. B. W. E. N. Mohak Chadha, "A statistical approach to power estimation for x86 processors," in 2017 IEEE International Parallel and Distributed Processing Symposium Workshops (IPDPSW), 2017.

[6]. U. Sirin, R. Appuswamy and A. Ailamaki, "OLTP on a server-grade ARM: power, throughput and latency comparison," DaMoN '16: Proceedings of the 12th International Workshop on Data Management on New Hardware, pp. 1-7, 2016.

[7]. P. Otto, M. Malik, N. Akhlaghi, R. Sequeira, H. Homayoun and S. Sikdar, "Power and performance characterization, analysis and tuning for energy-efficient edge detection on atom and ARM based platforms," in 2015 33rd IEEE International Conference on Computer Design (ICCD), 2015.

[8]. A. Pellegrini, N. Stephens, M. Bruce, Y. Ishii, J. Pusdesris, A. Raja, C. Abernathy, J. Koppanalil, T. Ringe, A. Tummala, J. Jalal, M. Werkheiser and A. Kona, "The Arm Neoverse N1 Platform: Building Blocks for the Next-Gen Cloud-toEdge Infrastructure SoC," IEEE Micro, vol. 40, no. 2, 2020.

[9]. L. V. Davi and A. D. S. N. A.-R. Sadeghi, "Gadge me if you can: secure and efficient adhoc instruction-level randomization for x86 and ARM," pp. 299-310, 2013.

[10]. G. M. V. S. S. S. L. M. S. A. P. R. a. M. S. G. Kristopher Keipert $\dagger$ "Energy-Efficient Computational Chemistry: Comparison of x86 and ARM Systems," in American Chemical Society, 2015.

[11]. Z. Ou, P. Bo and Y. Deng, "Energy- and CostEfficiency Analysis of ARM-Based Clusters," in The 12th IEEE/ACM International Symposium on Cluster, Cloud and Grid Computing (CCGrid'12), 2012.
[12]. K. Cho and H. Bahn, "A Cost Estimation Model for Cloud Services and Applying to PC Laboratory Platforms," 4 January 2020.

[13]. D. K. D. Kalaskar, S. Yadav and P. Dhumane, "Advance Model For Cost Reduction In Cloud Computing Environment," INTERNATIONAL JOURNAL OF SCIENTIFIC \& TECHNOLOGY RESEARCH, vol. 8, no. 11, November 2019.

[14]. L. Lupori, V. Rosario and E. Borin, "Towards a High-Performance RISC-V Emulator," in 2018 Symposium on High Performance Computing Systems (WSCAD), 2018.

[15]. "The rise of RISC - [Opinion]," IEEE Spectrum, vol. 55, no. 8, 2018.

[16]. M. Ling, X. Xu, G. Yushen and Z. Pan, in 2019 IEEE Pacific Rim Conference on Communications, Computers and Signal Processing (PACRIM), 2019.

[17]. D. Patterson, "Reduced Instruction Set Computers Then and Now," vol. 50, no. 12, 2017.

[18]. H. El-Aawar, "An application of complexity measures in addressing modes for CISC- and RISC-architectures," in 2008 IEEE International Conference on Industrial Technology, 2008.

[19]. A. A. Abudaqa, T. M. Al-Kharoubi, M. F. Mudawar and A. Kobilica, "Simulation of ARM and x86 microprocessors using in-order and out-of-order CPU models with Gem5 simulator," in 2018 5th International Conference on Electrical and Electronic Engineering (ICEEE), 2018.

[20]. Y. a. F. S. G. S. Nicholas, "A Survey and Analysis of SoC Platform Security in ARM, Intel and RISC-V Architecture," in 2020 IEEE 63rd International Midwest Symposium on Circuits and Systems (MWSCAS), 2020.

[21]. E. Christiansen, "RISCy business: The future of x86," 2020. 
Khushi Gupta et al Int. J. Sci. Res. Comput. Sci. Eng. Inf. Technol, May-June - 2021, 7 (3) : 619-631

[22]. B. Thompson, "Intel Problems," 19 January 2021.

[23]. D. Graham-Smith, "ARM vs Intel Processors: What's the Difference?," 29 May 2021.

\section{Cite this article as :}

Khushi Gupta, Tushar Sharma, "Changing Trends in Computer Architecture : A Comprehensive Analysis of ARM and x86 Processors", International Journal of Scientific Research in Computer Science, Engineering and Information Technology (IJSRCSEIT), ISSN : 2456-3307, Volume 7 Issue 3, pp. 619-631, May-June 2021. Available at doi : https://doi.org/10.32628/CSEIT2173188 Journal URL : https://ijsrcseit.com/CSEIT2173188 